$$
\begin{aligned}
& \text { IN } \\
& \text { Cos }
\end{aligned}
$$

NASA Technical Memorandum 107230

\title{
A Technique for Synergistic Atomic Oxygen and Vacuum Ultraviolet Radiation Durability Evaluation of Materials for use in LEO
}

Sharon K. Rutledge and Bruce A. Banks

Lewis Research Center

Cleveland, Ohio

Prepared for the

Third International Conference on Protection of Materials and Structures

From the Low Earth Orbit Space Environment

cosponsored by the Canadian Space Agency and

the Institute for Space and Terrestrial Studies

Toronto, Canada, April 25-26, 1996

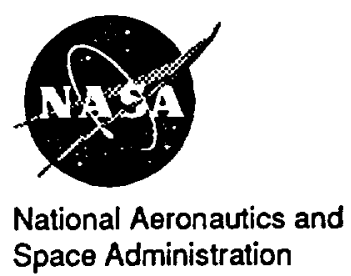


Trade names or manufacturers' names are used in this report for identification only. This usage does not constitute an official endorsement, either expressed or implied, by the National Aeronautics and Space Administration. 


\title{
A Technique for Synergistic Atomic Orygen and Vacuum Ultraviolet Radiation Durability Evaluation of Materials for Use in LEO
}

\author{
Sharon K. Rutledge \\ Bruce A. Banks \\ NASA Lewis Research Center \\ Cleveland, Ohio
}

\begin{abstract}
Material erosion data collected during flight experiments such as the Environmental Oxygen Interaction with Materials (EOIM)-III and the Long Duration Exposure Facility (LDEF) have raised questions as to the sensitivity of material erosion to levels of atomic oxygen exposure and vacuum ultraviolet (VUV) radiation. The erosion sensitivity of some materials such as FEP Teflon used as a thermal control material on satellites in low Earth orbit (LEO), is particularly important but difficult to determine. This is in large part due to the inability to hold all but one exposure parameter constant during a flight experiment. This is also difficult to perform in a ground based facility, because often the variation of the level of atomic oxygen or VUV radiation also results in a change in the level of the other parameter. A facility has been developed which allows each parameter to be changed almost independently and offer broad area exposure. The resulting samples can be made large enough for mechanical testing. The facility uses an electron cyclotron resonance plasma source to provide the atomic oxygen. A series of glass plates is used to focus the atomic oxygen while filtering the VUV radiation from the plasma source. After filtering, atomic oxygen effective flux levels can still be measured which are as high as $7 \times 10^{15}$ atoms $/ \mathrm{cm}^{2}-\mathrm{sec}$ which is adequate for accelerated testing. VUV radiation levels after filtering can be as low as 0.3 suns. Additional VUV suns can be added with the use of deuterium lamps which allow the VUV level to be changed while keeping the flux of atomic oxygen constant. This paper discusses the facility, and results from exposure of Kapton and FEP at pre-determined atomic oxygen flux and VUV sun levels.
\end{abstract}




\section{INTRODUCTION}

The qualification of materials for use in space is a challenging task. Testing of material degradation is most reliable when the material can be exposed to its' exact use environment. For materials used in LEO applications, exposure to the exact environment using a flight experiment is difficult to achieve. Long term effects of the LEO environment cannot be easily extrapolated from short duration Space Shuttle missions. Often the exposure is so short for some materials that changes in optical, thermal, physical or mechanical properties are not detectable within the error of the instruments being used to measure the effect. Longer duration free-flyer satellites such as LDEF$^{1}$ have provided some valuable long term exposure information, but these types of opportunities are infrequent and often the exposure conditions are not within the experimenter's control. Ground-based test facilities to simulate parts of the environment have been developed in order to provide designers with material degradation information that is more timely. These facilities when compared to flight experiments also have the advantages of lower cost per exposure and ease of tailorability to the specific environmental conditions of interest.

There are no ground-based facilities to date than can exactly duplicate all of the environmental conditions present in LEO. Many try to accurately simulate one part of the environment such as atomic oxygen, ultraviolet radiation, electrons, or protons. Even these individual mini-environments are difficult to simulate. Some of the most extensive work has been involved with simulating the atomic oxygen environment.

Atomic oxygen is the predominant constituent of the atmosphere between altitudes of $180-650 \mathrm{~km}^{2}$ It is created by the photodissociation of molecular oxygen by energetic solar photons. In LEO, the mean free path between atomic collisions is long enough that recombination of atomic oxygen into molecular oxygen is low. The passage of spacecraft through this environment produces a resulting collision with these atoms at energies on the order of $4.5 \mathrm{eV}^{3}$ This energy is very difficult to physically achieve on the ground. Most 
simulation facilities produce atomic oxygen which is either more energetic (10's to 100 's of $\mathrm{eV})$ or less energetic (0.04-0.1 eV) than this. Ground based facilities run the gambit from thermal plasmas to energetic directed beams. ${ }^{4567}$ Although some facilities can produce atomic oxygen at energies close to that in space, there are no known facilities to date which can duplicate the exact energy, species, and atomic states over a broad exposure area. Concerns about the effects of accelerated testing, the presence of ionic species, differences in energy and others have yet to be resolved, but ground based facilities represent a viable testing method for material qualification if the results can be correlated to material performance in LEO. There has been some investigation into theoretical models which can provide this correlation. ${ }^{8}$

This paper discusses one atomic oxygen facility, adapted to provide the ability to control synergistic VUV radiation to allow radiation dependent atomic oxygen reaction studies to take place. Beam characterization and the results of exposure of Kapton and FEP Teflon will be discussed.

\section{ATOMIC OXYGEN DIRECTED BEAM FACILITY}

In the Directed Beam Facility, atomic oxygen is produced inside a vacuum chamber $71 \mathrm{~cm}$ in diameter by $1.71 \mathrm{~m}$ long. Pumping is provided by a diffusion pump, mechanical pump and roots-type blower that operate on Fomblin (perfluorinated polyether) oil. The base pressure of the vacuum chamber is $2.7 \times 10^{-4} \mathrm{~Pa}\left(2 \times 10^{-6}\right.$ torr), but during operation can range from $0.027 \mathrm{~Pa}\left(2 \times 10^{-4}\right.$ torr $)$ to $0.107 \mathrm{~Pa}\left(8 \times 10^{-4}\right.$ torr $)$ depending on the oxygen gas flow rate.

The atomic oxygen is produced by an Electron Cyclotron Resonance (ECR) plasma source manufactured by Applied Science and Technology Inc. (ASTeX) operated on pure oxygen gas. The source operates at $2.45 \mathrm{GHz}$ with two electromagnets to dissociate molecular oxygen into atomic oxygen by energetic electron collisions. 
Microwave energy for the dissociation is absorbed by electrons at the cyclotron resonance condition created at a magnetic field of $\mathbf{8 7 5}$ gauss. The location of the region of plasma generation is determined as the location where the net magnetic field strength is equal to 875 gauss. A side view schematic of the source and vacuum chamber is shown in Figure 1.

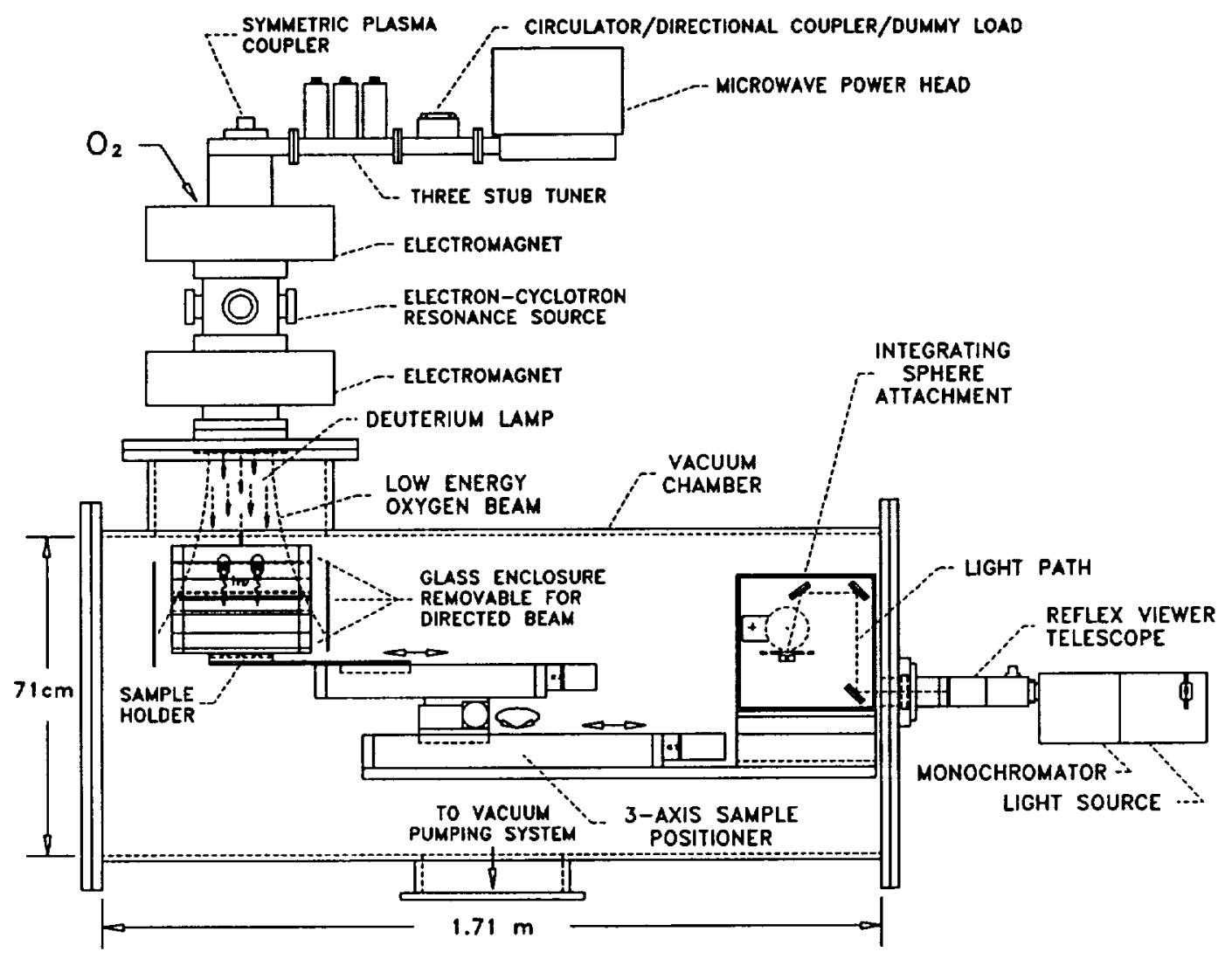

Figure 1. Side view schematic of vacuum chamber showing the ECR source, reflectance measurement system, motion arm, and VUV blocking apparatus (From Reference 4)

The upper electromagnet shown in the schematic is the predominant controller of the plasma formation location, which usually occurs between the two electromagnets. The lower electromagnet can shift this location or add a second plasma generation region if the current is high enough. The directed nature of the beam is driven by gas expansion from the higher pressure plasma formation region to the lower pressure sample region. This 
pressure difference can be 2-3 orders of magnitude. The lower magnet is used predominantly to steer the ions that are formed and will affect the flux distribution of both ions and atoms downstream. A quartz liner was installed between the two electromagnets to reduce recombination on the stainless steel chamber walls. Although the microwave power can be changed, the power was set at $1000 \mathrm{~W}$ for these tests.

The schematic also shows an integrating sphere internal to the vacuum chamber. The 3-axis sample positioner shown allows samples to be exposed to atomic oxygen or atomic oxygen in the presence of VUV radiation provided by Hammamatsu deuterium lamps. The samples can then be moved while still under vacuum to the integrating sphere where reflectance as a function of wavelength can be measured from 250 to $2500 \mathrm{~nm}$. The size of the facility allows test conditions to be flexible. For example, a furnace heater, a fiberglass tensioning mechanism, and a sample rotation device to simulate sweeping atomic oxygen arrival have been installed in this facility at various times. A programmable logic controller allows the system to be operated continuously without being attended. A multitude of safety interlocks and monitors allow the system to shut down in a safe manner should there be a power failure, microwave leak, loss of vacuum or other failure condition. A more complete description of this facility can be found in reference 4 . In addition to atomic oxygen, the ECR source generates intense $130 \mathrm{~nm}$ vacuum ultraviolet radiation produced during dissociation of the molecular oxygen. By using an Acton Research photomultiplier and filter wheel calibrated to a National Institute of Standards calibrated deuterium lamp, we were able to measure a VUV radiation intensity at $130 \mathrm{~nm}$ of approximately 150 suns. This level of VUV radiation can cause uncharacteristic damage to some UV sensitive materials. Therefore, it was important to reduce the VUV radiation to more acceptable levels without reducing the atomic oxygen flux below the $10^{15}$ atoms $/ \mathrm{cm}^{2}-\mathrm{sec}$ level needed to produce accelerated test results within a reasonable timeframe. 


\section{ATOMIC OXYGEN BEAM FACILITY CHARACTERIZATION}

\section{VUV Radiation Reduction from the Source}

\section{By Variation of Source Conditions:}

The level of VUV radiation can vary depending on the distance of the samples from the source and the location of the plasma formation region. For our testing, the sample distance from the source was fixed at $44.8 \mathrm{~cm}$ due to the location of the sample positioning arm, so there was no flexibility in this parameter. Changing the upper magnet current had a minimal effect on the VUV radiation intensity, but the lower magnet had a slight effect on intensity as measured by the photomultiplier. Lowering of the current on this magnet forced the ECR plasma generation region higher in the chamber thereby increasing the distance the radiation had to travel to reach the samples. The shift was not enough, however, to lower the VUV intensity below 144 suns at the lowest stable operating conditions for the source. This method of VUV radiation level reduction had several other drawbacks. Changing the operating conditions of the source also changed the atomic oxygen flux. This makes it impossible to compare changes in the erosion of a material due to variations in VUV radiation at a fixed atomic oxygen arrival rate. In addition, shifting the formation of the plasma higher into the chamber moved it just above the glass liner, where energetic electrons caused sputtering of the stainless steel walls. This caused deposits of iron and chromium to be observed by energy dispersive $\mathrm{x}$-ray analysis on the microwave guide window and in trace amounts on the samples.

\section{By Scattering Atomic Oxygen:}

Another technique to reduce VUV radiation is to confine the beam around a bend so that the samples are no longer line-of-sight to the plasma source. In this manner, VUV radiation cannot directly fall upon the samples, but the atomic oxygen flux can be reduced by interactions with the scattering surface and volume interactions. The beam contains so few ions, that a magnetic field would not be the most efficient way to bend the beam. So for the atomic beam, bouncing the atoms off of a low recombination, non-reactive, VUV transparent surface like fused silica seemed the best. In order to reduce losses of atoms 
during the bend, a fused silica pipe with a $90^{\circ}$ bend was fitted against the end of the quartz liner in the ECR source as shown in Figure 2. If a high enough flux could be achieved, then VUV could be added at the sample site with deuterium lamps. Unfortunately, the highest flux achievable was on the order of $2 \times 10^{14}$ atoms $/\left(\mathrm{cm}^{2}-\mathrm{sec}\right)$. The effective flux was determined by measuring the mass change of a polyimide Kapton sample placed at the end of the fused silica pipe. It was believed that the low flux level may have been caused by too many atomic collisions occurring in the higher pressure bend region and on the surface of the pipe which allowed the atomic oxygen to recombine to molecular oxygen.

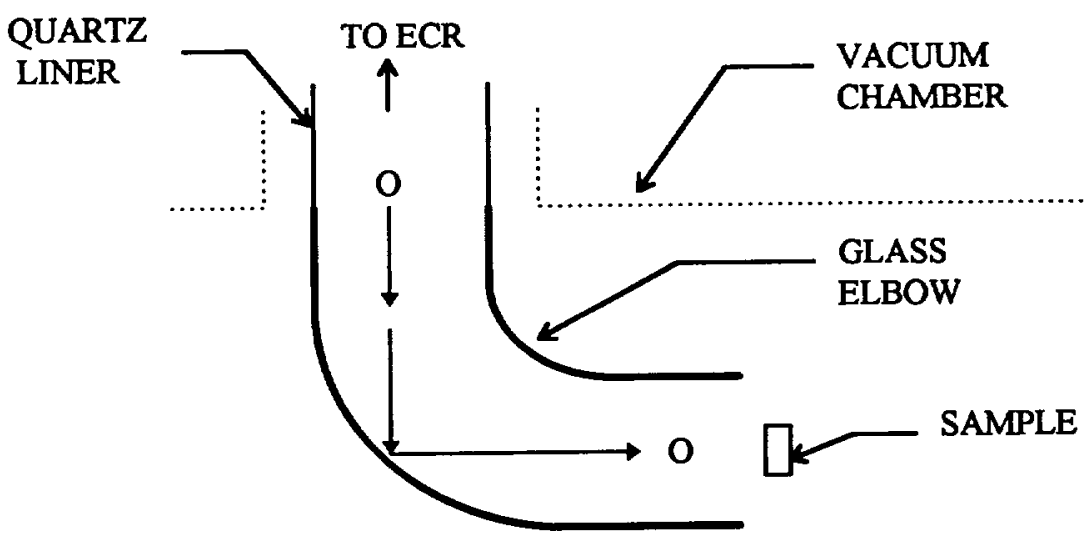

\section{Figure 2. Beam scattering apparatus design that will position samples away from direct VUV radiation arrival from the source}

In order to increase the flux, it was decided to try to minimize the bend that the beam would have to go through. Instead of moving away from the VUV, reducing the radiation level from the source was attempted by filtering. The chevron design shown in Figure 3 allowed atomic oxygen to scatter off of glass strips arranged in a pattern somewhat like a double venetian blind. The use of window glass instead of fused silica provided filtering of the VUV radiation by absorption of more of the VUV by the glass. It was thought that the atomic oxygen would scatter through the louvers and provide a 
ENLARGED

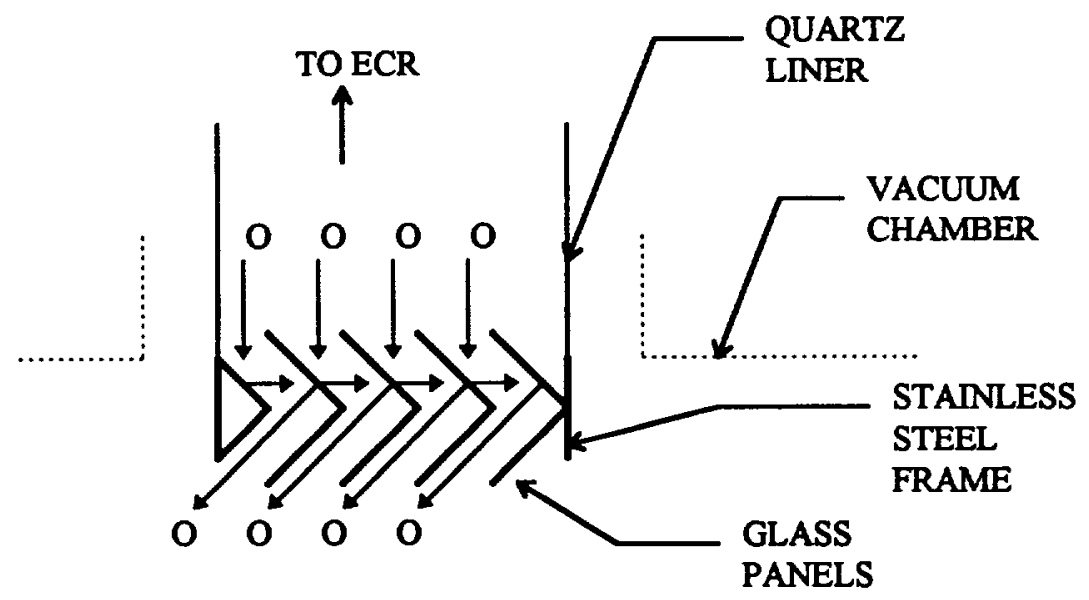

KAPTON

ETCH

PATTERN

\section{KAPTON SHEET}

Figure 3. Chevron design for filtering VUV radiation from the samples

diffuse beam of atomic oxygen to the samples. The flux level measured at the $44.8 \mathrm{~cm}$ sample plane was much higher on the average with this design (about $3 \times 10^{15}$ atoms $/ \mathrm{cm}^{2}$ sec), but the arrival was very non-uniform. The Kapton samples used for the flux measurement exhibited very distinct stripes of degradation (evidenced by surface texturing) that were about $1.9 \mathrm{~cm}$ wide. In ray tracing back to the chevron, it was discovered that these stripes corresponded to atoms traveling directly down the lower chevron channel which was a little over $2 \mathrm{~cm}$ wide. There was some evidence of scattering from the sides of the glass. However, degradation exhibited in regions reached by scattered atoms was much lower. The design of the louvers indicated that the first bounce off of the glass was most likely specular with the second bounce giving more of a cosine distribution.

From our previous testing, it appeared that the atomic oxygen scattering patterns from the chevrons were similar to those observed in light scattering. A design was prepared using ray tracing in order to provide blockage of VUV radiation while focusing the atomic oxygen to the sample plane. This resulted in the final design which is shown in 


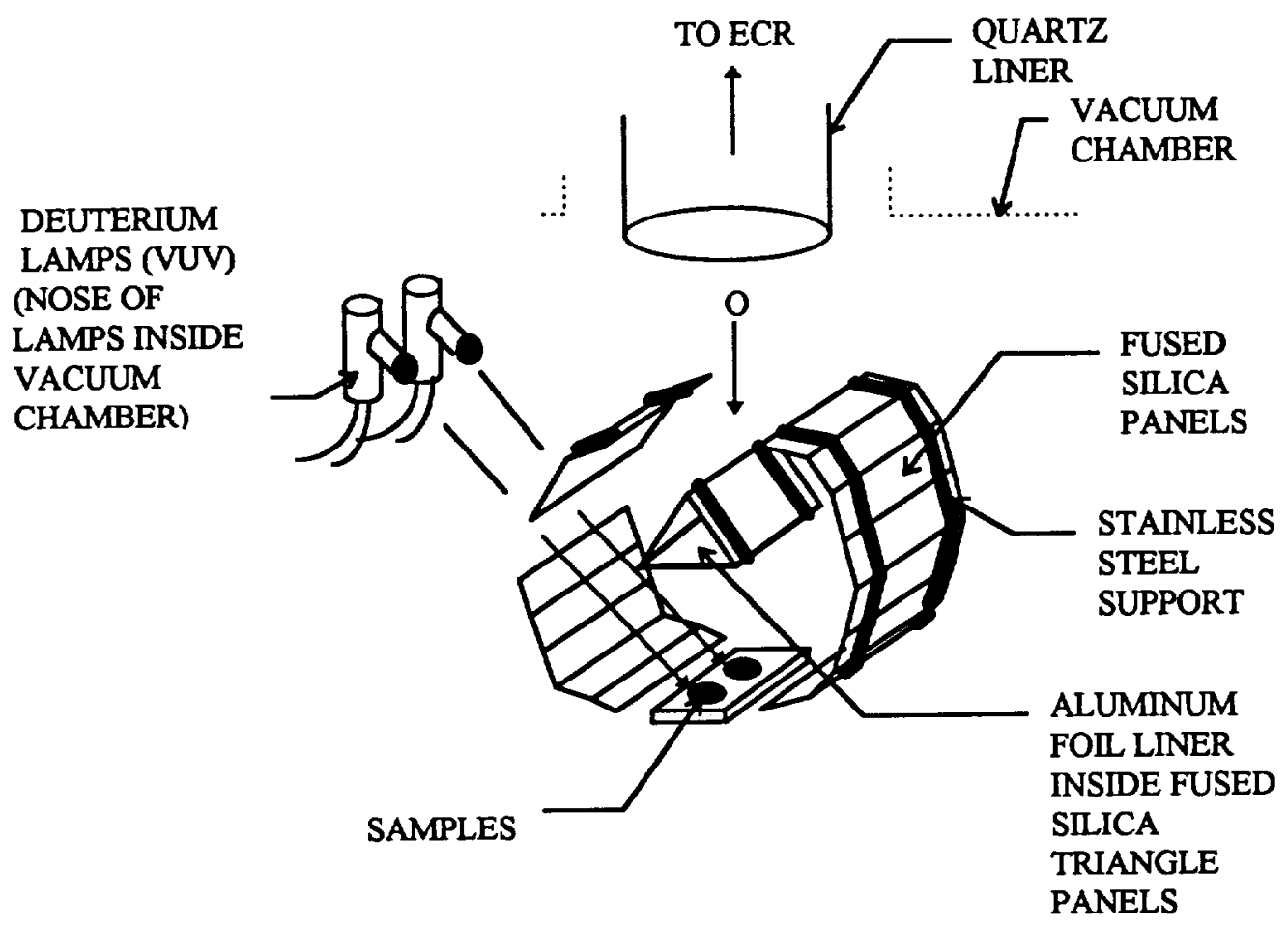

Figure 4. Atomic oxygen scattering and VUV blocking apparatus design that successfully produced a moderate atomic oxygen flux level while shielding out VUV radiation from the source

Figure 4 and Figure 1. The exact shape of the fused silica panels was determined by making a mock-up of the design from cardboard with a reflecting white surface. This mock-up was tested by putting a light source at the plasma formation plane and a solar cell at the sample position. The panels were moved until a maximum current reading was obtained on the solar cell. The actual atomic oxygen scattering system was constructed from stainless steel back supports with fused silica panels held to it with spot welded stainless steel straps. The triangle in the center was also made of fused silica panels held in place on each end with spot welded stainless straps. A sheet of $0.0127 \mathrm{~cm}$ thick aluminum foil was used to line the interior of the bottom of this open triangle in order to block VUV from the source from reaching the samples. The VUV level at the sample plane after installation of the hardware was found to range from 0.3 to 2 suns depending on the current settings for the lower magnet. Higher magnet currents resulted in higher VUV 
radiation levels because the plasma formation region is forced closer to the sample plane. This alters the line-of-sight path of the radiation from the source. Atomic oxygen effective fluxes measured at the sample plane using polyimide Kapton witness samples were in the $10^{15}$ atom/ $\left(\mathrm{cm}^{2}-\mathrm{sec}\right)$ range. A plot of the atomic oxygen flux as a function of spatial position for this source both with and without the focusing system is shown in Figure 5. For the flux mapping, the source was kept at $1000 \mathrm{~W}$ with oxygen gas flow of $50 \mathrm{SCCM}$ (standard cubic centimeters per minute) and upper and lower magnet currents of 182 and 125 amps respectively. In both cases, there is a fairly even distribution over the area of interest. Flux distribution typically will stay fairly uniform, but the overall levels can vary by changing the operating conditions of the source. As can be seen from Figure 5b, the flux level is about 3-5 times lower with the VUV block in place. There is also a slightly lower atomic oxygen flux underneath the fused silica triangle. One of the advantages of this design is that VUV radiation can be added from the side with deuterium lamps so that tests with both high and low levels of VUV can be run at the same atomic oxygen flux level.

\section{Energy and Ion Contribution}

Reference 4 describes characterization of the ions present in the beam from the ECR source both with the beam focusing apparatus and without. The source was characterized for these parameters at the same source operating conditions present when measuring the flux distribution. Without the VUV radiation block and atomic oxygen focusing apparatus, the percentage of ions in the beam was calculated to be near $0.55 \%$. The ion energy measured with a retarding potential analyzer, also discussed in reference 4, was a double lobed gaussian distribution with ion energy peaks at 10.5 and $25 \mathrm{eV}$. With the focusing and blocking apparatus installed, the ion population was $0.4 \%$ with the same source operating conditions. The ion energy in this case was a single peaked gaussian centered at $7 \mathrm{eV}$. It is believed that the ion energy is reduced in this case due to collisions with the glass surfaces. The uncharged atomic oxygen is believed to have an energy of approximately $0.04 \mathrm{eV}$. Reference 4 estimates that the percentage of Kapton mass loss 


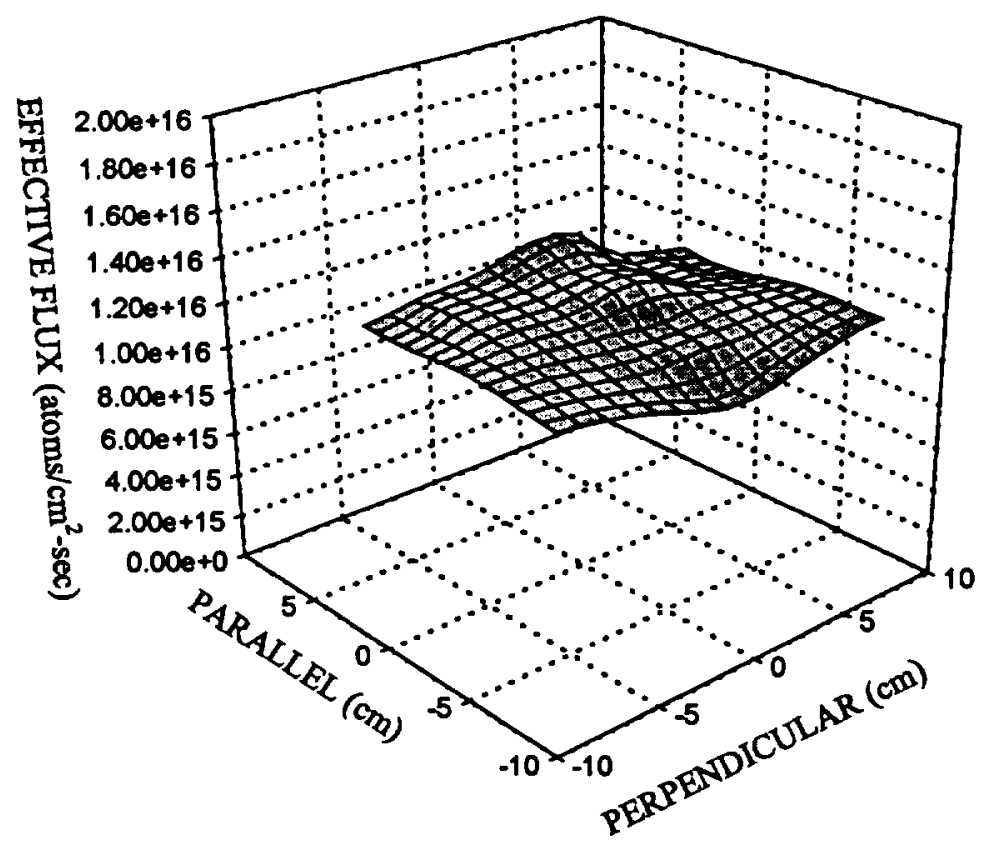

Figure 5a. Flux map for the ECR source with samples directly exposed to the beam

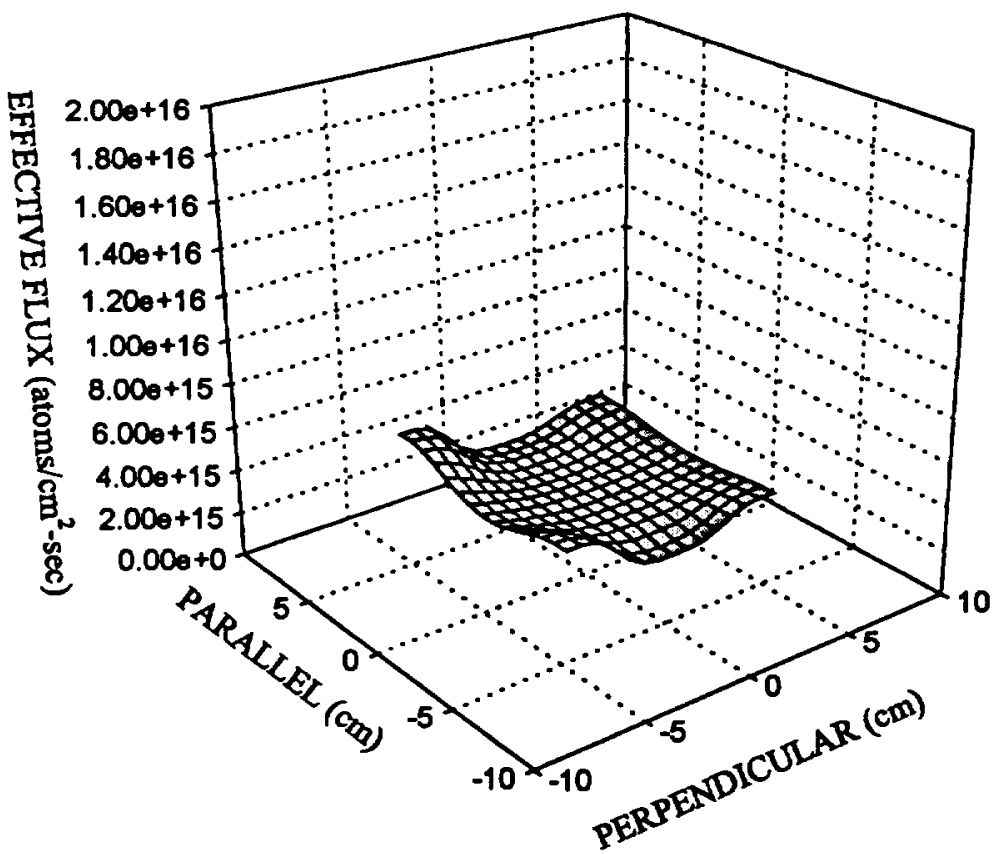

Figure 5b. Flux map for the ECR source with VUV blocking apparatus in place 
due to ions alone is less than $15 \%$ in both cases. Therefore, most of the material loss should be attributable to thermal energy atoms.

\section{KAPTON AND FEP TEFLON EXPOSURE}

\section{Preparation and Analysis Techniques}

\section{Test Design:}

Three factors have been thought to play the greatest role in affecting the erosion yield of FEP. They are VUV radiation intensity, atomic oxygen flux and atomic oxygen fluence. These three factors were made to be controllable and largely independent through the addition and removal of the VUV blocking apparatus and deuterium lamps. This allowed testing to be performed to shed some light on FEP erosion yield sensitivity to each of these parameters independently.

Due to the complexity and time limitations placed on each test, it was necessary to carefully select the exposure conditions in order to achieve the most information for the minimum amount of testing. The Taguchi model for Design of Experiments ${ }^{9}$ was used to determine the most informative series of tests to perform in the directed beam facility. Samples of FEP and Kapton were exposed at the same time so that the Kapton could be used to determine the effective flux and fluence levels of atomic oxygen present.

Temperature may also have an influence, but it was not a controllable factor during these tests so it was only monitored. Because testing time was limited, two levels (high and low) were selected for each factor. An L8 factorial design was used. Level selection was limited to the high and low ranges achievable in the vacuum facility. Where possible, additional data was included from other tests. 


\section{Sample Preparation and Dehydration:}

FEP Teflon and Kapton HN of approximately $0.005 \mathrm{~cm}$ in thickness supplied by DuPont were cut into $2.54 \mathrm{~cm}$ diameter disks with a punch for exposure. All of the samples were fully dehydrated in vacuum and weighed prior to and after exposure in order to minimize errors in mass due to water absorption. ${ }^{10}$ Typically, samples are removed from a vacuum of 8-13 $\mathrm{Pa}$ (60-100 mTorr) and then four weight measurements are made within five minutes on a Mettler M3 microbalance. These weight measurements are averaged and recorded along with the standard deviation. Most change in mass measurement errors are on the order of $2 \times 10^{-5}$ to $2 \times 10^{-4} \mathrm{~g}$ when measurements are made in this manner. Weighing the same piece of Kapton, after removal from vacuum, at recorded time intervals for twelve separate trials and extrapolating the mass of the Kapton back to time equal to zero still results in an error of $1.24 \times 10^{-5} \mathrm{~g}$. This appears to be the best that can be achieved when limited by drift in the instrument. This represents a $0.5 \%$ error in mass measurement for low fluences (near $10^{20}$ atoms $/ \mathrm{cm}^{2}$ ). This is still much lower than the $8-10 \%$ error that can be experienced without sample dehydration at low fluence levels.

\section{Data Measurement and Analysis:}

The effective fluence for each exposure was derived from the Kapton exposure data. Using the known erosion yield for Kapton in low Earth orbit $\left(3 \times 10^{-24} \mathrm{~cm}^{3} / a\right.$ tom), equation 1 can be used to solve for the effective fluence, knowing the density of Kapton and the measured mass loss per unit area. The erosion yield for Kapton is reported to vary with the energy of the arriving oxygen atoms ${ }^{4}$ but the models in the literature only agree on the erosion yield for $4.5 \mathrm{eV}$ LEO atoms. So if the actual fluence were needed, the energy dependence would have to be known. The effective fluence, however, is not an absolute measure of atoms per unit area arriving at the surface, it is the calculation of the equivalent atoms per unit area in LEO which would produce the same damage as observed in the ground based facility and is strictly used as a means of comparison. 


$$
F=\frac{d M}{A \rho E} \quad \text { Equation 1 }
$$

Where: $F=$ Effective Fluence for Kapton (atoms $/ \mathrm{cm}^{2}$ )

$d M=$ Change in Mass of Kapton (g)

$A=$ Exposed Surface Area $\left(\mathrm{cm}^{2}\right)$

$\rho=$ Density of Kapton $\left(\mathrm{g} / \mathrm{cm}^{3}\right)$

$E=$ Erosion Yield for Kapton ( $\left.\mathrm{cm}^{3} / \mathrm{atom}\right)$

The erosion yield of a material with respect to the erosion yield of Kapton, however, can be determined without knowing the fluence if they were both exposed together to the same atomic oxygen arrival. In this case, equation 2 can be used to give the erosion yield ratio for the material of interest using Kapton as the reference because fluence drops out of the equation.

$$
\begin{gathered}
\frac{E_{M}}{E_{K}}=\frac{d M_{M} A_{K} \rho_{K}}{d M_{K} A_{M} \rho_{M}} \quad \text { Equation } 2 \\
\text { Where: } E_{M}=\text { Erosion Yield of the Material }\left(\mathrm{cm}^{3} / \mathrm{atom}\right) \\
E_{K}=\text { Erosion Yield of Kapton }\left(\mathrm{cm}^{3} / \text { atom }\right) \\
d M_{K}=\text { Change in Mass of Kapton }(\mathrm{g}) \\
d M_{M}=\text { Change in Mass of the Material }(\mathrm{g}) \\
A_{K}=\text { Exposed Surface Area of Kapton }\left(\mathrm{cm}^{2}\right) \\
A_{M}=\text { Exposed Surface Area of the Material }\left(\mathrm{cm}^{2}\right) \\
\rho_{K}=\text { Density of Kapton }\left(\mathrm{g} / \mathrm{cm}^{3}\right) \\
\rho_{M}=\text { Density of the Material }\left(\mathrm{g} / \mathrm{cm}^{3}\right)
\end{gathered}
$$

In order to determine if Kapton effective flux, Kapton effective fluence, or VUV radiation intensity contributed to the relative erosion yield, a regression model was fit to the data in order to look for significant trends and the amount of error that could be explained by each factor. The computer program that was used for the regression model and analysis of variance (ANOVA) was STORM ${ }^{11}$. In the statistical analysis, R-squared values indicate goodness of fit of the computer generated model with the data. R-squared 
values range from 0 to 1.0 with 1.0 being a perfect fit. Two statistical measures were used to indicate the significance of a particular factor. The " $F$ " value is the measure of the mean square error due to the factor of interest divided by the mean square error due to all other factors. It is an indication of the extent to which the spread observed in the data can be explained by a particular factor. The probability or "p-value" is also an indicator of the same thing but it represents the probability that the factor is not significant. P-values of $<0.05(5 \%)$ are considered to be statistically significant.

\section{$\underline{\text { Results and Discussion }}$}

Source conditions during exposure, levels of the three independent factors, and the erosion yield of FEP Teflon with respect to Kapton are shown in Table I for each test performed. The atomic oxygen effective flux levels, effective fluence levels, and VUV intensity levels were controlled by changing the magnet current, time of exposure, and installing or removing the VUV blocking apparatus with or without addition of deuterium lamps.

FEP Teflon has been thought to be sensitive to VUV radiation effects due to the potential for VUV induced crosslinking to take place. The data from the directed beam, however, indicates that this may not be the case for the VUV intensity levels used in these tests. For all of the tests performed with 50 SCCM oxygen gas flow, similar relative erosion yields were obtained at the same effective flux and effective fluence levels whether the samples were exposed to 0.3 suns or 150 suns. Regression analysis indicates that for these intensity levels, the probability of relative erosion yield dependence for FEP Teflon on VUV intensity is very low. The F-value was 0.13 ( $p=0.73$ for insignificance).

Regression analysis for the 50 SCCM oxygen gas flow data gives fairly strong support for a slight relative erosion yield dependence on fluence. This change may be due to the development of a surface texture and resultant increase in surface area with 
Table I. Test Results for ECR Exposure of FEP Teflon

\begin{tabular}{|c|c|c|c|c|c|c|c|c|}
\hline Power (W) & $\begin{array}{l}\text { Sample } \\
\text { Temperature } \\
\left.\text { ( }{ }^{\circ} \mathrm{C}\right)\end{array}$ & $\begin{array}{l}\text { Upper } \\
\text { Magnet } \\
\text { Current } \\
\text { (Amps) }\end{array}$ & $\begin{array}{l}\text { Lower } \\
\text { Magret } \\
\text { Curremt } \\
\text { (Amps) }\end{array}$ & $\begin{array}{l}\text { Gas } \\
\text { Flow } \\
\text { (SCCM) }\end{array}$ & $\begin{array}{l}\text { UV } \\
\text { Radiation } \\
\text { Intensity at } \\
130 \mathrm{~nm} \\
\text { (auns) }\end{array}$ & $\begin{array}{l}\text { Effective } \\
\text { Atomic } \\
\text { Oxygen } \\
\text { Fluence } \\
\text { (aloms/ } / \mathrm{cm}^{2} \text { ) }\end{array}$ & $\begin{array}{l}\text { Effective } \\
\text { Alomic } \\
\text { Oxygen } \\
\text { Flux } \\
\text { (atoms/cm². } \\
\text { ecc) }\end{array}$ & $\begin{array}{l}\text { Erotion } \\
\text { Yield of } \\
\text { FEP Teflon } \\
\text { Relative } \\
\text { to Kapton }\end{array}$ \\
\hline 1000 & $<41$ & 150 & 30 & 50 & 0.3 & 1.1E20 & $1.1 \mathrm{E} 15$ & $1.2 \pm 0.5$ \\
\hline 1000 & $<41$ & 150 & 30 & 50 & 0.3 & 4.4E20 & $9.2 \mathrm{E} 14$ & $1.5 \pm 0.1$ \\
\hline 1000 & $<41$ & 150 & 125 & 50 & 1.8 & $1.2 \mathrm{E} 20$ & 7.2E15 & $1.2 \pm 0.1$ \\
\hline 1000 & $<41$ & 150 & 125 & 50 & 1.8 & $3.9 \mathrm{E} 20$ & $5.4 \mathrm{E} 15$ & $1.6 \pm 0.1$ \\
\hline 1000 & $<41$ & 180 & 125 & 70 & $1-1.5$ & $1.8 \mathrm{E} 20$ & $4.9 \mathrm{E} 15$ & $2.5 \pm 0.3$ \\
\hline 1000 & $<41$ & 180 & 125 & 70 & $4-5$ & $1.8 \mathrm{E} 20$ & $4.9 \mathrm{E} 15$ & $2.6 \pm 0.3$ \\
\hline 1000 & $<41$ & 180 & 125 & 70 & $1-1.5$ & $5.6 \mathrm{E} 20$ & $4.5 \mathrm{E} 15$ & $2.2 \pm 0.2$ \\
\hline 1000 & $<41$ & 180 & 125 & 70 & $4-5$ & $5.7 \mathrm{E} 20$ & 4.7E15 & $2.2 \pm 0.2$ \\
\hline 1000 & $<41$ & 180 & 30 . & 30 & 145 & $27 E 20$ & $5.0 \mathrm{E} 15$ & $1.2 \pm 0.1$ \\
\hline 1000 & $<41$ & 150 & 30 & 50 & 144 & $2,1 \mathrm{E} 20$ & $422 \mathrm{E} 15$ & $1.4 \pm 0.1$ \\
\hline 1000 & 143. & 180 & 125 & So & 150 & $3.7 \mathrm{E} 20$ & $1.6 \mathrm{E} 16$ & $1,5 \pm 0.1$ \\
\hline 1000 & 143 & 180 & 125 & 50 & 150 & $1.1 E 20$ & $1.6 \mathrm{E} 16$ & $1.2 \pm 0.1$ \\
\hline 1000 & 143 & 180 & 125 & 30 & 150 & $4.3 \mathrm{E} 20$ & $2.0 \mathrm{E} 16$ & $1.6+0.1$ \\
\hline 1000 & 143 & 180 & 125 & 50 & 150 & 4.3E20 & $1.9 \mathrm{E} 16$ & $1.6 \pm 0.1$ \\
\hline
\end{tabular}

* Shaded entries were made without VUV blocking apparatus in place

effective fluence. Evidence of surface texturing was observed by scanning electron microscopy and by eye as an increase in diffuse reflectance. Up to a limit, increased surface area could afford multiple opportunities for incoming atomic oxygen to react through partial trapping. The F-value for this factor was $38.06(p=0.00027)$. Of the three factors studied, this was the only significant factor. The F-value for flux dependence was $2.22(p=0.18)$. The $R$-squared value for the overall fit was 0.83 . Although temperature was not included in the model, it did not appear to play a role between $41^{\circ} \mathrm{C}$ and $143^{\circ} \mathrm{C}$.

Four tests were conducted at 70 SCCM oxygen gas flow but were not part of the design model. The results of these tests are interesting because they differ greatly from those performed at $50 \mathrm{SCCM}$. One effect of raising the oxygen gas flow appears to be a limiting of the effective flux. The flux appears to be limited to approximately $5 \times 10^{15}$ atoms $/\left(\mathrm{cm}^{2}-\mathrm{sec}\right)$, while at $50 \mathrm{SCCM}$, higher flux conditions can be achieved with similar source conditions (flux levels are affected primarily by changes in lower magnet current when the same physical configuration is used). It is possible that the increased gas flow results in more oxygen atom collisions. This could both reduce the atomic oxygen flux by 
recombination and possibly lower the ion energy through these same collision processes. Even though less than $15 \%$ of the mass loss can be explained by ion interaction with the Kapton, a change in ion energy could shift the mass loss and resulting effective flux somewhat if the erosion yield dependence on energy is great. Kapton also seemed to be insensitive to VUV radiation between 1 and 5 suns because the same erosion was observed for the same exposure time both with the deuterium lamps on and off.

Another effect observed was a near doubling of the FEP erosion yield with respect to that for Kapton at 70 SCCM gas flow. Again, there was no observed change due to VUV radiation between 1 and 5 suns for all of the tests conducted at 70 SCCM oxygen gas flow. At this level, there was also not a significant change in erosion yield ratio with effective fluence over the range of fluences tested. Erosion yield ratios measured at similar effective flux and effective fluence levels at 50 SCCM still were nearly half the erosion yield ratios obtained at 70 SCCM. Why is there a difference? There is currently no firm explanation. There could be a change in the erosion yield of FEP with respect to Kapton for changing ion energies. No change in the relative erosion yield for FEP was observed between the $7 \mathrm{eV}$ ion arrival observed with the VUV blocking apparatus and the double peaked energy distribution at 10.5 and $25 \mathrm{eV}$ observed with no VUV block in place. However, it is possible that by further decreasing the energy, the erosion of Kapton may reduce more than that of FEP resulting in a higher relative erosion yield for FEP with respect to Kapton.

The relative erosion yield for FEP with respect to Kapton as measured on a sample cut from the same material lot and flown on the EOIM-III experiment aboard the U.S. Space Shuttle (STS-46) was $0.06 \pm 0.01$. This sample received an effective fluence of $2.6 \times 10^{20}$ atoms $/ \mathrm{cm}^{2}$ at an effective flux of $1.8 \times 10^{15}$ atoms $/ \mathrm{cm}^{2}-\mathrm{sec}$, and VUV intensity of 0.2 suns. These levels are not very different from those used in the directed beam exposure, yet the relative erosion yield is about 20 times higher for the samples exposed in the ground laboratory directed beam compared to that in LEO at similar VUV intensity, effective fluence, and effective flux conditions. The fact that these numbers are so different 
provides strong evidence that there is another factor which affects the erosion yield of FEP in relation to Kapton in the directed beam that has not been considered. Some possibilities in addition to energy are ion population, soft $\mathrm{X}$-rays, charged species, metastables or other unknown factors. Until the factor which affects the erosion yield is found, materials with an FEP component will need to have an FEP witness coupon rather than a Kapton one to determine the effective erosion yield.

\section{CONCLUSIONS}

Atomic oxygen with various levels of synergistic VUV has been able to be produced over a broad area to enable studies of critical factors for erosion dependencies to take place. By the addition of a VUV blocking apparatus, and addition of deuterium lamps, levels of VUV intensity ranging from 0.3 to 150 suns could be produced while keeping atomic oxygen effective flux and effective fluence values nearly constant. Exposure of FEP Teflon to a matrix of exposure conditions, and concentrating on the factors of effective flux, effective fluence and VUV intensity, allowed the significance of

these factors to be determined. It was found that the erosion yield of FEP with respect to Kapton is not sensitive to VUV radiation intensity over the range tested. It did not appear to be sensitive to effective flux either, but did change slightly with effective fluence. The data obtained at a different gas flow rate for oxygen and space data for FEP indicates that there are still changes in the relative erosion yield that cannot be explained by the three factors that were studied. Other factors present in the facility will need to be investigated for their effect. Until the factor or factors are found that produce these changes, when testing materials of a particular group such as FEP Teflon, it may be best to use a witness sample made of a similar material that already has some space data available. This would enable one to predict an equivalent exposure in the ground based facility. Comparisons of this type between ground based facilities and space enables materials to be effectively screened on the ground where exposure and number of samples exposed can be better tailored to meet the types of information needed. 


\section{ACKNOWLEDGMENTS}

The authors would like to thank Joyce Dever at the NASA Lewis Research Center for providing measurements of the vacuum ultraviolet radiation intensity in the atomic oxygen directed beam. Also thank you to Michael Cales of Cleveland State University for measuring the minimum error for Kapton mass measurements.

\section{REFERENCES}

${ }^{1}$ Clark, L.G.; Kinard, W.H.; Carter, D.J.; and Jones, J.L.; "Long Duration Exposure Facility (LDEF) Mission I Experiments", NASA SP 473, 1984.

${ }^{2}$ United States Committee on Extension to the Standard Atmosphere, U.S. Standard Atmosphere, 1976 U.S. Government Printing Office, Washington, D.C., 1976.

${ }^{3}$ Banks, B.A.; deGroh, K.K.; Rutledge, S.K.; and DiFilippo, F.J.; "Prediction of In-Space Durability of Protected Polymers Based on Ground Laboratory Thermal Energy Atomic Oxygen Testing"; ICPMSE-3 Conference, Toronto, Canada; April 25-26, 1996.

${ }^{4}$ Stidham, C.R et al; "Low Earth Orbit Atomic Oxygen Environmental Simulation Facility for Space Materials Evaluation", NASA TM-106128, 1993.

s Johnson, C.L.; et al. "The Vanderbilt University Neutral O-Beam Facility"; 18th International SAMPE Technical Conference Proceedings, Oct. 7-9, 1986.

${ }^{6}$ Brinza, D.E.; et al.; "Production of Pulsed Atomic Oxygen Beams Via Laser Vaporization Methods"; 18th International SAMPE Technical Conference Proceedings, Oct. 7-9, 1986.

'Caledonia, G.E.; et al.; "A High Flux Source of Energetic Oxygen Atoms for Material Degradation Studies"; AIAA 85-7015.

${ }^{8}$ Banks, B.A.; deGroh, K.K.; Bucholz, J.L. and Cales, M.R.; “Atomic Oxygen Interactions with Protected Organic Materials on the Long Duration Exposure Facility (LDEF)"; Proceedings of the Third Long Duration Exposure Facility (LDEF) Conference; Williamsburg, VA, Nov. 8-12, 1993.

${ }^{9}$ Montgomery, D.C.; Design and Analysis of Experiments, 3rd Edition, John Wiley and Sons, 1991.

${ }^{10}$ Rutledge, S.K.; et al. "An Evaluation of Candidate Atomic Oxygen Resistant Materials for Space Applications in LEO", NASA TM-100122, 1986.

"Emmons, H.; et al. STORM, Quantitative Modeling for Decision Support; Version 3.0; Storm Software Inc., 1992. 


\begin{tabular}{|c|c|c|c|c|}
\hline \multicolumn{3}{|c|}{ REPORT DOCUMENTATION PAGE } & & $\begin{array}{l}\text { Form Approved } \\
\text { OMB No. 0704-0188 }\end{array}$ \\
\hline \multicolumn{5}{|c|}{ 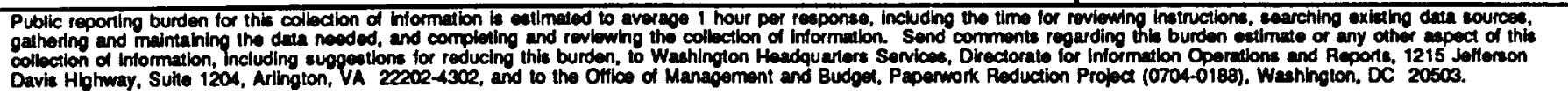 } \\
\hline 1. AGENCY USE ONLY (Leave blank) & \begin{tabular}{r|r} 
2. REPOAT DATE \\
May 1996
\end{tabular} & \multicolumn{3}{|c|}{$\begin{array}{l}\text { 3. REPORT TYPE AND DATES COVERED } \\
\text { Technical Memorandum }\end{array}$} \\
\hline \multicolumn{4}{|c|}{\begin{tabular}{l|l} 
4. TILE AND SUBTIRLE & 5. F \\
A Technique for Synergistic Atomic Oxygen and Vacuum Ultraviolet Radiation & \\
Durability Evaluation of Materials for use in LEO &
\end{tabular}} & \\
\hline \multicolumn{3}{|c|}{$\begin{array}{l}\text { 6. AUTHOR(S) } \\
\text { Sharon K. Rutledge and Bruce A. Banks }\end{array}$} & & \\
\hline \multicolumn{3}{|c|}{$\begin{array}{l}\text { 7. PERFORMING ORGANIZATION NAME(S) AND ADDRESS(ES) } \\
\text { National Aeronautics and Space Administration } \\
\text { Lewis Research Center } \\
\text { Cleveland, Ohio } 44135-3191\end{array}$} & \multicolumn{2}{|c|}{$\begin{array}{l}\text { 8. PERFORMING ORGANIZATION } \\
\text { REPORT NUMBER }\end{array}$} \\
\hline \multicolumn{3}{|c|}{ 9. SPONSORINGMONITORING AGENCY NAME(S) AND ADDRESS(ES) } & 10. SP & $\begin{array}{l}\text { NSORINGMONTTORING } \\
\text { NCY REPORT NUMBER }\end{array}$ \\
\hline \multicolumn{5}{|c|}{$\begin{array}{l}\text { 11. SUPPLEMENTARY NOTES } \\
\text { Prepared for the Third International Conference on Protection of Materials and Structures From the Low Earth Orbit Space } \\
\text { Environment cosponsored by the Canadian Space Agency and the Institute for Space and Terrestrial Studies, Toronto, } \\
\text { Canada, April 25-26, 1996. Responsible person, Sharon K. Rutledge, organization code 5480, (216) 433-2219. }\end{array}$} \\
\hline \multicolumn{4}{|c|}{\begin{tabular}{l|l} 
12a. DISTRIBUTLONAVAILABILITY STATEMENT & 12b. \\
Unclassified - Unlimited \\
Subject Category 23 \\
This publication is available from the NASA Center for AeroSpace Information, (301) 621-0390.
\end{tabular}} & 2b. DISTAIBUTION CODE \\
\hline \multicolumn{5}{|c|}{ 13. ABSTRACT (Maximum 200 words) } \\
\hline \multicolumn{5}{|c|}{$\begin{array}{l}\text { Material erosion data collected during flight experiments such as the Environmental Oxygen Interaction with Materials } \\
\text { (EOIM)-III and the Long Duration Exposure Facility (LDEF) have raised questions as to the sensitivity of material erosion } \\
\text { to levels of atomic oxygen exposure and vacuum ultraviolet (VUV) radiation. The erosion sensitivity of some materials } \\
\text { such as FEP Teflon used as a thermal control material on satellites in low Earth orbit (LEO), is particularly important but } \\
\text { difficult to determine. This is in large part due to the inability to hold all but one exposure parameter constant during a } \\
\text { flight experiment. This is also difficult to perform in a ground based facility, because often the variation of the level of } \\
\text { atomic oxygen or VUV radiation also results in a change in the level of the other parameter. A facility has been developed } \\
\text { which allows each parameter to be changed almost independently and offer broad area exposure. The resulting samples can } \\
\text { be made large enough for mechanical testing. The facility uses an electron cyclotron resonance plasma source to provide } \\
\text { the atomic oxygen. A series of glass plates is used to focus the atomic oxygen while filtering the VUV radiation from the } \\
\text { plasma source. After filtering, atomic oxygen effective flux levels can still be measured which are as high as } 7 x 10^{15} \text { atoms/ } \\
\mathrm{cm}^{2} \text {-sec which is adequate for accelerated testing. VUV radiation levels after filtering can be as low as } 0.3 \text { suns. Additional } \\
\text { VUV suns can be added with the use of deuterium lamps which allow the VUV level to be changed while keeping the flux } \\
\text { of atomic oxygen constant. This paper discusses the facility, and results from exposure of Kapton and FEP at pre-deter- } \\
\text { mined atomic oxygen flux and VUV sun levels. }\end{array}$} \\
\hline \multicolumn{3}{|l|}{ 14. SUBJECT TERMS } & & $\begin{array}{l}\text { 15. NUMBEA OF PAGES } \\
21\end{array}$ \\
\hline \multicolumn{4}{|c|}{ Atomic oxygen; Flux; Fluence; VUV radiation; Durability; Erosion; Yield; FEP } & $\begin{array}{l}\text { 16. PRICE CODE } \\
\text { A03 }\end{array}$ \\
\hline $\begin{array}{l}\text { 17. SECURTY CLASSIFICATION } \\
\text { OF REPORT } \\
\text { Unclassified }\end{array}$ & $\begin{array}{l}\text { 18. SECURTY CLASSIFICATION } \\
\text { OF THIS PAGE } \\
\text { Unclassified }\end{array}$ & $\begin{array}{l}\text { 19. SEC } \\
\text { OF }\end{array}$ & ITION & 20. LIMITATION OF ABSTRACT \\
\hline
\end{tabular}




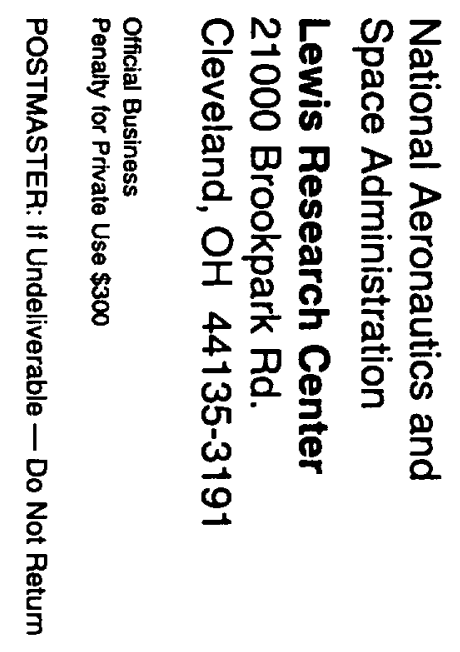

